\title{
Microarray analysis of microRNA deregulation and angiogenesis-related proteins in endometriosis
}

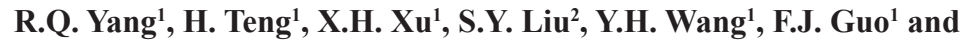 \\ X.J. Liu $^{3}$ \\ ${ }^{1}$ Department of Obstetrics and Gynecology, \\ The Second Hospital of Jilin University, Changchun, Jilin Province, China \\ ${ }^{2}$ Department of Anesthesia, The Second Hospital of Jilin University, \\ Changchun, Jilin Province, China \\ ${ }^{3}$ Department of Pathology, The First Hospital of Jilin University, \\ Changchun, Jilin Province, China \\ Corresponding author: X.J. Liu \\ E-mail: xuej_liu@sina.com
}

Genet. Mol. Res. 15 (2): gmr.15027826

Received October 28, 2015

Accepted February 12, 2016

Published June 3, 2016

DOI http://dx.doi.org/10.4238/gmr.15027826

\begin{abstract}
We examined the aberrant microRNA (miRNA) expression profile responsible for the changes in angiogenesis observed in endometriotic lesions. This study revealed characteristic miRNA expression profiles associated with endometriosis in endometrial tissue and endometriotic lesions from the same patient, and their correlation with the most important angiogenic and fibrinolytic factors. miRNA expression was quantified using a microRNA array and reversetranscription microRNA polymerase chain reaction. Levels of vascular endothelial growth factor A (VEGFA), epidermal growth factor receptor 2 (EGFR2), phosphatase and tensin homolog (PTEN), and $\mathrm{C}-\mathrm{X}-\mathrm{C}$ chemokine receptor type 4 (CXCR4) were quantified using enzyme-linked immunosorbent assay. The endometrial tissue showed significantly lower levels of miR-200b, miR-15a-5p, miR-19b-1-5p, miR-146a-5p, and miR-200c, and higher levels of miR-16-5p, miR106b-5p, and miR-145-5p. VEGFA was significantly upregulated,
\end{abstract}


whereas EGFR2, PTEN, and CXCR4 were markedly downregulated, in the endometriotic tissues compared to that in the normal endometrial tissues. In conclusion, differences in the miRNA levels could modulate the expression of VEGFA, EGFR2, PTEN, and CXCR4, and may play an important role in the pathogenesis of endometriosis. The higher angiogenic and proteolytic activities observed in the eutopic endometrium might facilitate the implantation of endometrial cells at ectopic sites.

Key words: Endometriosis; MicroRNA; VEGFA; EGFR2; PTEN; CXCR4

\section{INTRODUCTION}

Endometriosis is a frequent, chronic, benign disease that is characterized by the growth of proliferative endometrial-like tissue outside the uterus. It commonly affects the ovary, uterosacral ligament, recto-uterine pouch, and the uterine serosa, causing pelvic pain and even infertility. Endometriosis affects $5-10 \%$ of all women of reproductive age, especially from age 25 to 45 , and the prevalence rises to $20-50 \%$ in infertile women (Taylor et al., 2009). Although endometriosis is considered benign, it is associated with an increased risk of malignant transformation in approximately $1.0 \%$ of affected women, with the involvement of multiple pathways of development (Forte et al., 2014). However, the etiology and pathogenesis of endometriosis are still unclear.

microRNAs (miRNAs) are short, noncoding single-stranded RNA molecules that can regulate gene expression. Since miRNAs were first discovered, they have been identified as significant mediators in cellular physiology and pathology. Recently, studies have demonstrated that aberrant miRNA expression is strongly linked with tumor-related processes in humans (Fassan et al., 2011; Li et al., 2011; Manavalan et al., 2011; Matsuyama et al., 2011). Moreover, miRNAs appear to be potent regulators of gene expression in endometriosis and its associated reproductive disorders, raising the prospect of using miRNAs as biomarkers and therapeutic tools in endometriosis (Teague et al., 2010). miR-191 modulates malignant transformation in endometriosis by regulating TIMP3 (Dong et al., 2015), and inhibits the TNF- $\alpha$-induced apoptosis of ovarian endometriosis and endometrioid carcinoma cells by targeting DAPK1 (Tian et al., 2015). Aberrantly expressed miR-20a contributes to endometriosis by decreasing NTN4 expression (Zhao et al., 2014). In addition, as with tumor metastasis, endometriosis is an angiogenesis-dependent disease, and endometriotic implants require neovascularization to proliferate and invade ectopic sites within the host (Taylor et al., 2009). Research has shown that endometrial miR-200c targets the expression of ZEBs, VEGFA, FLT1, IKK $\beta$, KLF9, and FBLN5, and then influences cellular angiogenesis, transformation, and inflammation during normal and endometriotic progression (Panda et al., 2012).

The present study was designed to examine the miRNA expression profile in endometriotic tissues compared with normal human endometrium tissues. Reversetranscription (RT) miRNA polymerase chain reaction (PCR) and miRNA microarray analysis revealed aberrant miRNA expression in the endometriotic tissues. We also determined the characteristic miRNA expression profiles associated with endometriosis in endometrial tissue and endometriotic lesions from the same patient, and their correlation with the most important angiogenic and fibrinolytic factors. 


\section{MATERIAL AND METHODS}

\section{Endometriotic tissues and normal controls}

Endometriotic tissues were obtained from 32 female patients with endometriosis (stages III to IV) diagnosed by laparoscopic findings according to the revised American Fertility Society classification of endometriosis guidelines (American Society for Reproductive Medicine, 1997). Control endometrial tissues were obtained from 19 premenopausal patients who had undergone hysterectomies for subserosal leiomyoma without evidence of endometriosis, as described in a previous study (Adachi et al., 2011). All the women had abstained from medication for at least 3 months prior to donating the tissues. The use of each specimen was formally allowed with written consent from each subject before the study, which was approved by the Ethics Committee of the Second Hospital of Jilin University. All the tissues were homogenized and incubated in TRIzol reagent (Invitrogen, Carlsbad, CA, USA) before storage at $-80^{\circ} \mathrm{C}$.

\section{miRNA microarray analysis}

Total RNAs were extracted from the TRIzol reagent-dissolved tissues using a miRVana miRNA Isolation Kit (Ambion, Austin, TX, USA) according to the manufacturer instructions, and were quantified using a NanoDrop ND-1000 spectrophotometer (NanoDrop Technologies, Wilmington, DE, USA). miRNA samples then were hybridized to Affymetrix GeneChip miRNA arrays using a FlashTag Biotin RNA labeling kit (Genisphere, Hatfield, PA, USA) according to the manufacturer instructions. The microarray message was analyzed using Feature Extraction v.9.5.3.1 (Agilent, Santa Clara, CA, USA).

\section{Real-time PCR analysis for miRNA}

Each miRNA sample was first reverse-transcribed with a Multiplex RT pool set, and was then quantified with a mirVana ${ }^{\mathrm{TM}}$ reverse transcription-quantitative real-time PCR (RT-qPCR) miRNA detection kit (Thermo Scientific, Rockford, IL, USA) according to the manufacturer instructions. Specific primer pairs for miR-106b-5p, miR-126, miR-142, miR145-5p, miR-146a-5p, miR-148a, miR-150, miR-15a-5p, miR-16-5p, miR-181a, miR-19b1-5p, miR-200b, miR-200c, miR-205, miR-423, miR-663, miR-675, and miR-21-5p were utilized for each target miRNA. Real-time PCR was performed on a StepOnePlus ${ }^{\mathrm{TM}}$ system (Applied Biosystems, Foster City, CA, USA) using the following conditions: heat activation of reverse DNA polymerase at $95^{\circ} \mathrm{C}$ for $10 \mathrm{~min}$, followed by 40 cycles of $95^{\circ} \mathrm{C}$ for $15 \mathrm{~s}$, and $60^{\circ} \mathrm{C}$ for $1 \mathrm{~min}$. Small nuclear U6 RNA was taken as a control, and the $\Delta \Delta \mathrm{Ct}$ method was used for relative quantification (Livak and Schmittgen, 2001).

\section{Enzyme-linked immunosorbent assay (ELISA)}

The levels of vascular endothelial growth factor A (VEGFA), epidermal growth factor receptor 2 (EGFR2), phosphatase and tensin homolog (PTEN), and C-X-C chemokine receptor type 4 (CXCR4) in the samples from each endometriotic tissue or the control were determined using an ELISA kit (Westang Bio, Shanghai, China), according to the manufacturer instructions. Each homogenized tissue sample was centrifuged for $30 \mathrm{~min}$ at $9400 \mathrm{~g}$ and $4^{\circ} \mathrm{C}$, and the supernatant was collected. Serially diluted standard samples or endometriotic tissue 
samples $(100 \mu \mathrm{L})$ were added to the ELISA microplate and incubated at $37^{\circ} \mathrm{C}$ for $2 \mathrm{~h}$, and then $100 \mu \mathrm{L}$ diluted antibody against VEGFA, EGFR2, PTEN, or CXCR4 was added to the plate for further incubation at $37^{\circ} \mathrm{C}$ for $1 \mathrm{~h}$. Finally, the plate was incubated with $100 \mu \mathrm{L}$ horseradish peroxidase-conjugated secondary antibody for $30 \mathrm{~min}$ at $37^{\circ} \mathrm{C}$, and with $100 \mu \mathrm{L}$ substrate in the dark for $15 \mathrm{~min}$. Each well was washed three times with $100 \mu \mathrm{L}$ phosphate-buffered saline with Tween before each inoculation. The specific binding optical density of each well was determined immediately at $450 \mathrm{~nm}$.

\section{Statistical analysis}

All quantitative data are reported as means \pm standard error of the mean. Statistical analyses were performed using the Student $t$-test for comparisons of two groups, and $\mathrm{P}$ values $<0.05$ were considered statistically significant.

\section{RESULTS}

\section{miRNA profiling by microarray analysis in endometriotic tissues}

To screen the specific expression of miRNAs in endometrium and/or endometriotic tissues, miRNA profiling by microarray was performed on five endometriotic tissue samples and five control endometrium specimens. As shown in Table 1, there were 17 miRNAs with microarray signaling. Moreover, the microarray analysis indicated that there were three miRNAs with an upregulation of more than 1.5-fold: 3.154-fold for miR-145-5p, 2.735-fold for miR-106b-5p, and 2.471-fold for miR-16-5p in the endometriotic tissues compared with the normal endometrium tissues. However, miR-200b, miR-15a-5p, miR-19b-1-5p, and miR-146a$5 \mathrm{p}$ were downregulated by 1.931 -fold, 1.704 -fold, 1.536 -fold, and 1.522-fold, respectively.

Table 1. miRNA microarray expression in the endometrium of women without endometriosis and in the endometriotic tissues of women with endometriosis.

\begin{tabular}{l|c|c|c}
\hline \multirow{2}{*}{ Gene symbol } & \multicolumn{2}{|c|}{ Microarray value } & Fold-change \\
\cline { 2 - 4 } & Control $(\mathrm{N}=5)$ & Endometriotic tissues (N =5) & \\
\hline miR-106b-5p & 0.1116 & 0.3052 & 2.735 \\
\hline miR-126 & 0.3150 & 0.2668 & 0.847 \\
\hline miR-142 & 0.2085 & 0.2504 & 1.201 \\
\hline miR-145-5p & 0.1203 & 0.3793 & 3.154 \\
\hline miR-146a-5p & 0.1098 & 0.0721 & 0.657 \\
\hline miR-148a & 0.0353 & 0.0312 & 0.885 \\
\hline miR-150 & 0.1683 & 0.1508 & 0.896 \\
\hline miR-15a-5p & 0.1422 & 0.0835 & 0.587 \\
\hline miR-16-5p & 0.1059 & 0.2617 & 2.471 \\
\hline miR-181a & 0.1932 & 0.2376 & 0.140 \\
\hline miR-19b-1-5p & 0.2280 & 0.1484 & 0.651 \\
\hline miR-200b & 0.1125 & 0.0583 & 0.518 \\
\hline miR-200c & 0.1510 & 0.1145 & 0.763 \\
\hline miR-205 & 0.2067 & 0.2286 & 1.106 \\
\hline miR-663 & 0.2229 & 0.2189 & 0.982 \\
\hline miR-675 & 0.1374 & 0.1841 & 0.340 \\
\hline
\end{tabular}

miRNA levels in endometrium and endometriotic tissues compared with the normal controls

To confirm the up- or downregulation of the miRNAs mentioned above, real-time 
PCR analysis was performed with specific pared primers for each target miRNA in 19 control endometrium specimens and 32 endometriotic specimens. The relative quantitative analysis indicated that four miRNAs in the 32 endometriotic tissues were significantly upregulated (miR-106b-5p, miR-145-5p, miR-16-5p, and miR-21-5p); the relative levels of the four upregulated miRNAs were $2.10 \pm 0.24,3.62 \pm 0.43,1.87 \pm 0.21$, and $2.84 \pm 0.31$, respectively $(\mathrm{P}<0.001$ or $\mathrm{P}<0.0001$; Table 2$)$. In contrast, six miRNAs were markedly downregulated in the endometriotic tissues (miR-126, miR-146a-5p, miR-15a-5p, miR-19b-1-5p, miR-200b, and miR-200c) $(\mathrm{P}<0.05, \mathrm{P}<0.01$, or $\mathrm{P}<0.001$; Table 2). These results demonstrate the aberrant miRNA expression profile in the endometriotic tissues.

Table 2. miRNA levels in the endometriotic tissues of women with endometriosis relative to those in the endometria of women without endometriosis.

\begin{tabular}{l|c|c|c}
\hline \multirow{2}{*}{ Gene symbol } & \multicolumn{2}{|c|}{ Endometrium } & \multirow{2}{*}{ Significance } \\
\cline { 2 - 4 } & Control $(\mathrm{N}=19)$ & Endometriotic tissues $(\mathrm{N}=32)$ & \\
\hline miR-106b-5p & $1.00 \pm 0.11$ & $2.10 \pm 0.24$ & $* * *$ \\
\hline miR-126 & $1.00 \pm 0.09$ & $0.76 \pm 0.08$ & $* *$ \\
\hline miR-145-5p & $1.00 \pm 0.13$ & $3.62 \pm 0.43$ & $* .50 \pm 0.06$ \\
\hline miR-146a-5p & $1.00 \pm 0.13$ & $0.67 \pm 0.06$ & $* *$ \\
\hline miR-15a-5p & $1.00 \pm 0.12$ & $1.87 \pm 0.21$ & $* *$ \\
\hline miR-16-5p & $1.00 \pm 0.08$ & $0.73 \pm 0.06$ & $* *$ \\
\hline miR-200b & $1.00 \pm 0.09$ & $0.62 \pm 0.07$ & $* *$ \\
\hline miR-200c & $1.00 \pm 0.11$ & $0.81 \pm 0.09$ & $*$ \\
\hline miR-21-5p & $1.00 \pm 0.09$ & $2.84 \pm 0.31$ & $* *$ \\
\hline
\end{tabular}

$* \mathrm{P}<0.05, * * \mathrm{P}<0.01, * * * \mathrm{P}<0.001, * * * * \mathrm{P}<0.0001$.

\section{Expression of angiogenesis-related proteins}

In addition, to explore the possible target markers for miRNAs in the endometriotic tissues, we then screened the homologous sequences in the untranslated region of possible protein markers with the miRNAs mentioned above. The possible target proteins for the 10 markedly regulated miRNAs are listed in Table 3. In particular, angiogenesis-related proteins such as VEGFA, EGFR2, PTEN, and CXCR4 were most frequently recognized as possible targets for these miRNAs in the endometriotic tissues. ELISA was conducted to determine the levels of these protein markers in endometriotic tissues. As shown in Figure 1, the results indicated that VEGFA was significantly upregulated and EGFR2, PTEN, and CXCR4 were markedly downregulated in the endometriotic tissues compared with the normal endometrial tissues.

Table 3. miRNAs expressed in the microarrays and targets of the miRNAs selected for the polymerase chain reaction (PCR) experiments.

\begin{tabular}{|c|c|c|}
\hline miRNA & miRNA sequence (5'-3') & Targets \\
\hline $\operatorname{miR}-19 b-1-5 p$ & aguuuugcagguuugcauccagc & 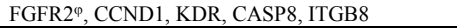 \\
\hline miR-200b-3p & uaauacugccugguaaugauga & 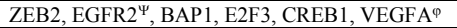 \\
\hline miR-16-5p & uagcagcacguaaauauuggeg & 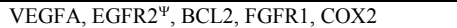 \\
\hline miR-126-3p & ucguaccgugaguaauaaugcg & VEGFA, CRK, SPRED1, PIK3R2, IRS1 \\
\hline miR-15a-5p & uagcagcacauaaugguuugug & $\mathrm{BCL}^{\varphi}$, VEGFA, CCND1, CCNE1, MYB, CCND2 \\
\hline miR-106b-5p & uaaagugcugacagugcagau & CDKN1A, VEGFA $^{\varphi}$, E2F1, RB1, PTEN, STAT3 \\
\hline miR-200c-3p & uaauacugccggguaaugaugga & ZEB1, BMI1, VEGFA, FN1, FBLN5 \\
\hline $\operatorname{miR}-21-5 p$ & uagcuuaucagacugauguuga & PDCD4, PTEN ${ }^{\Psi}$, RPS7, RECK, EFNA3, BMPR2 \\
\hline miR-146a-5p & ugagaacugaauuccauggguu & $\mathrm{CXCR}^{\Psi}{ }^{\Psi}, \mathrm{IRAK} 1, \mathrm{TRAF}^{\Psi}, \mathrm{EGFR}^{4}{ }^{\Psi}, \mathrm{BRCA} 1$ \\
\hline miR-145-5p & guccaguuuucccaggaaucccu & IRS1, CBFB, FSCN1, KLF4, MUC1, EGFR2 ${ }^{\Psi}$ \\
\hline
\end{tabular}

${ }^{\varphi}$ Possible targets for the downregulated miRNAs; ${ }^{\Psi}$ Possible targets for the upregulated miRNAs. 
A

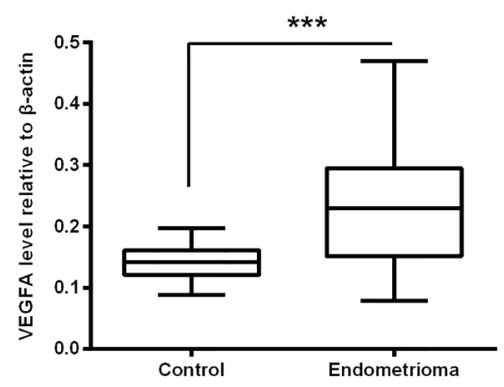

C

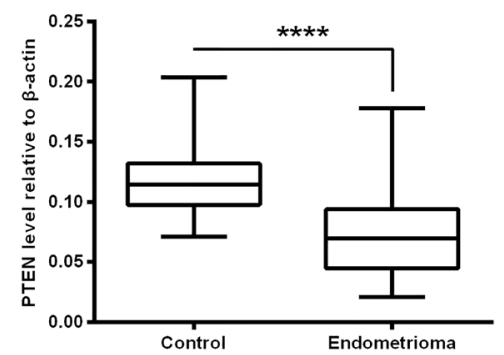

B

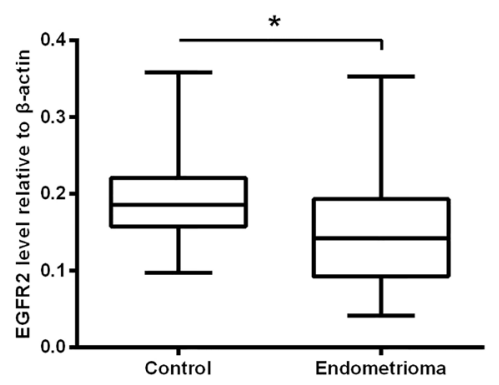

D

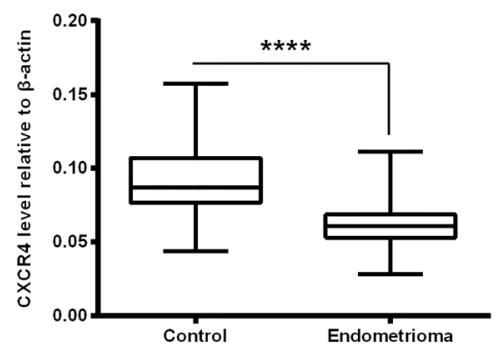

Figure 1. miRNA expression of angiogenesis-related proteins. The levels of vascular endothelial growth factor A (VEGFA), epidermal growth factor receptor 2 (EGFR2), phosphatase and tensin homolog (PTEN), and C-X-C chemokine receptor type 4 (CXCR4) were quantified by enzyme-linked immunosorbent assay (ELISA). A.-D. Expression of angiogenesis-related proteins (VEGF-A, EGFR2, PTEN, and CXCR4) compared with the control group and relative to $\beta$-actin. Statistical significance shown as $* \mathrm{P}<0.05,{ }^{*} * \mathrm{P}<0.001$, and $* * * * \mathrm{P}<0.0001$.

\section{DISCUSSION}

miRNAs are small (approximately 22 nucleotides in length), noncoding singlestranded RNA molecules that can regulate numerous biological processes, including cellular differentiation (Nolte-'t Hoen et al., 2015), proliferation (Zhao et al., 2012), apoptosis (Yeh et al., 2012), angiogenesis (Caporali and Emanueli, 2011), the development of cancer (Liu et al., 2015), and the inhibition of tumor angiogenesis and growth (Luo et al., 2013; Tu et al., 2015). Endometriosis is a common, although mysterious and fascinating, gynecological condition with diverse clinical manifestations and high variability. It has an unpredictable clinical course and results in diminished quality of life (Okeke et al., 2011). Despite extensive research, the pathogenesis of endometriosis remains unclear because it is complex. Recently, several studies have revealed that aberrant miRNA profiles are correlated with endometriosis (Ohlsson Teague et al., 2009; Braza-Boïls et al., 2014; Rekker et al., 2015). In particular, our own and previous studies indicated that members of the miR-200 family (miR-200a, miR-200b, and miR-200c) were downregulated in endometriosis-associated tissues (Ohlsson Teague et al., 2009; Braza-Boïls et al., 2014; Rekker et al., 2015). However, Saare et al. (2014) found that miR-200b was upregulated in such tissues. In addition, miR-20a plays an important role in the pathogenesis of ovarian endometriosis by suppressing NTN4 (Zhao et al., 2014), miR23a 
and miR23b are potential biomarkers of ovarian endometriosis (Shen et al., 2013), and miR$196 \mathrm{~b}$ targets c-myc and Bcl-2 expression, inhibiting proliferation and inducing apoptosis in endometriotic stromal cells (Abe et al., 2013).

The current study was conducted to detect aberrant miRNA expression in endometriotic tissues compared with normal endometrium tissues. Using a microRNA array and RT microRNA PCR, we found four upregulated miRNAs and six downregulated miRNAs in the endometriotic tissues (Tables 1 and 2). Furthermore, we explored the correlation between angiogenesis-related proteins (VEGFA, EGFR2, PTEN, and CXCR4) and endometriotic tissues using ELISA. Compared with the normal controls, the level of VEGFA was significantly increased, and the levels of EGFR2, PTEN, and CXCR4 were reduced in the endometriotic tissues (Figure 1). As listed in Table 3, the (5'-3') sequences of these miRNAs, and their targets in the endometriotic tissue samples were examined by PCR experiments. In particular, VEGF gene polymorphisms are thought to contribute to the risk of endometriosis (Li et al., 2013). We speculated that the deregulated miRNAs might regulate VEGF, thereby contributing to the pathogenesis of endometriosis.

In summary, we found aberrant expression of certain miRNAs in endometriotic tissues compared with those in normal endometrium tissues. We provided evidence that angiogenic and proteolytic activities, and VEGFA, EGFR2, PTEN, and CXCR4 expression levels were modulated by differentially expressed miRNAs in the endometriotic tissue samples. These findings may promote the use of miRNAs as biomarkers and therapeutic tools for endometriosis, and may provide important insight into the pathogenesis of endometriosis.

\section{Conflicts of interest}

The authors declare no conflict of interest.

\section{REFERENCES}

Abe W, Nasu K, Nakada C, Kawano Y, et al. (2013). miR-196b targets c-myc and Bcl-2 expression, inhibits proliferation and induces apoptosis in endometriotic stromal cells. Hum. Reprod. 28: 750-761. http://dx.doi.org/10.1093/humrep/ $\underline{\operatorname{des} 446}$

Adachi M, Nasu K, Tsuno A, Yuge A, et al. (2011). Attachment to extracellular matrices is enhanced in human endometriotic stromal cells: a possible mechanism underlying the pathogenesis of endometriosis. Eur. J. Obstet. Gynecol. Reprod. Biol. 155: 85-88. http://dx.doi.org/10.1016/j.ejogrb.2010.10.026

American Society for Reproductive Medicine (1997). Revised American Society for Reproductive Medicine classification of endometriosis: 1996. Fertil. Steril. 67: 817-821. http://dx.doi.org/10.1016/S0015-0282(97)81391-X

Braza-Boïls A, Marí-Alexandre J, Gilabert J, Sánchez-Izquierdo D, et al. (2014). MicroRNA expression profile in endometriosis: its relation to angiogenesis and fibrinolytic factors. Hum. Reprod. 29: 978-988. http://dx.doi. org/10.1093/humrep/deu019

Caporali A and Emanueli C (2011). MicroRNA regulation in angiogenesis. Vascul. Pharmacol. 55: 79-86. http://dx.doi. org/10.1016/j.vph.2011.06.006

Dong M, Yang P and Hua F (2015). MiR-191 modulates malignant transformation of endometriosis through regulating TIMP3. Med. Sci. Monit. 21: 915-920. http://dx.doi.org/10.12659/MSM.893872

Fassan M, Croce CM and Rugge M (2011). miRNAs in precancerous lesions of the gastrointestinal tract. World J. Gastroenterol. 17: 5231-5239. http://dx.doi.org/10.3748/wjg.v17.i48.5231

Forte A, Cipollaro M and Galderisi U (2014). Genetic, epigenetic and stem cell alterations in endometriosis: new insights and potential therapeutic perspectives. Clin. Sci. 126: 123-138. http://dx.doi.org/10.1042/CS20130099

Li XM, Wang AM, Zhang J and Yi H (2011). Down-regulation of miR-126 expression in colorectal cancer and its clinical significance. Med. Oncol. 28: 1054-1057. http://dx.doi.org/10.1007/s12032-010-9637-6 
Li YZ, Wang LJ, Li X, Li SL, et al. (2013). Vascular endothelial growth factor gene polymorphisms contribute to the risk of endometriosis: an updated systematic review and meta-analysis of 14 case-control studies. Genet. Mol. Res. 12: 1035-1044. http://dx.doi.org/10.4238/2013.April.2.20

Liu S, Su W, Li Z and Ding X (2015). Electrochemical detection of lung cancer specific microRNAs using 3D DNA origami nanostructures. Biosens. Bioelectron. 71: 57-61. http://dx.doi.org/10.1016/j.bios.2015.04.006

Livak KJ and Schmittgen TD (2001). Analysis of relative gene expression data using real-time quantitative PCR and the $2^{-\Delta \Delta C t}$ method. Methods 25: 402-408. http://dx.doi.org/10.1006/meth.2001.1262

Luo C, Tetteh PW, Merz PR, Dickes E, et al. (2013). miR-137 inhibits the invasion of melanoma cells through downregulation of multiple oncogenic target genes. J. Invest. Dermatol. 133: 768-775. http://dx.doi.org/10.1038/ jid.2012.357

Manavalan TT, Teng Y, Appana SN, Datta S, et al. (2011). Differential expression of microRNA expression in tamoxifensensitive MCF-7 versus tamoxifen-resistant LY2 human breast cancer cells. Cancer Lett. 313: 26-43. http://dx.doi. org/10.1016/j.canlet.2011.08.018

Matsuyama H, Suzuki HI, Nishimori H, Noguchi M, et al. (2011). miR-135b mediates NPM-ALK-driven oncogenicity and renders IL-17-producing immunophenotype to anaplastic large cell lymphoma. Blood 118: 6881-6892. http:// dx.doi.org/10.1182/blood-2011-05-354654

Nolte-'t Hoen EN, Van Rooij E, Bushell M, Zhang CY, et al. (2015). The role of microRNA in nutritional control. $J$. Intern. Med. 278: 99-109. http://dx.doi.org/10.1111/joim.12372

Ohlsson Teague EM, Van der Hoek KH, Van der Hoek MB, Perry N, et al. (2009). MicroRNA-regulated pathways associated with endometriosis. Mol. Endocrinol. 23: 265-275. http://dx.doi.org/10.1210/me.2008-0387

Okeke TC, Ikeako LC and Ezenyeaku CC (2011). Endometriosis. Niger. J. Med. 20: 191-199.

Panda H, Pelakh L, Chuang TD, Luo X, et al. (2012). Endometrial miR-200c is altered during transformation into cancerous states and targets the expression of ZEBs, VEGFA, FLT1, IKK $\beta$, KLF9, and FBLN5. Reprod. Sci. 19: 786-796. http://dx.doi.org/10.1177/1933719112438448

Rekker K, Saare M, Roost AM, Kaart T, et al. (2015). Circulating miR-200-family micro-RNAs have altered plasma levels in patients with endometriosis and vary with blood collection time. Fertil. Steril. 104: 938-946.e2. http://dx.doi. org/10.1016/j.fertnstert.2015.06.029

Saare M, Rekker K, Laisk-Podar T, Sõritsa D, et al. (2014). High-throughput sequencing approach uncovers the miRNome of peritoneal endometriotic lesions and adjacent healthy tissues. PLoS One 9: e112630. http://dx.doi.org/10.1371/ journal.pone. 0112630

Shen L, Yang S, Huang W, Xu W, et al. (2013). MicroRNA23a and microRNA23b deregulation derepresses SF-1 and upregulates estrogen signaling in ovarian endometriosis. J. Clin. Endocrinol. Metab. 98: 1575-1582. http://dx.doi. org/10.1210/jc. $2012-3010$

Taylor RN, Yu J, Torres PB, Schickedanz AC, et al. (2009). Mechanistic and therapeutic implications of angiogenesis in endometriosis. Reprod. Sci. 16: 140-146. http://dx.doi.org/10.1177/1933719108324893

Teague EM, Print CG and Hull ML (2010). The role of microRNAs in endometriosis and associated reproductive conditions. Hum. Reprod. Update 16: 142-165. http://dx.doi.org/10.1093/humupd/dmp034

Tian X, Xu L and Wang P (2015). MiR-191 inhibits TNF- $\alpha$ induced apoptosis of ovarian endometriosis and endometrioid carcinoma cells by targeting DAPK1. Int. J. Clin. Exp. Pathol. 8: 4933-4942.

Tu Y, Liu L, Zhao D, Liu Y, et al. (2015). Overexpression of miRNA-497 inhibits tumor angiogenesis by targeting VEGFR2. Sci. Rep. 5: 13827. http://dx.doi.org/10.1038/srep13827

Yeh CH, Chen TP, Wang YC, Lin YM, et al. (2012). MicroRNA-27a regulates cardiomyocytic apoptosis during cardioplegia-induced cardiac arrest by targeting interleukin 10-related pathways. Shock 38: 607-614. http://dx.doi. org/10.1097/SHK.0b013e318271f944

Zhao M, Tang Q, Wu W, Xia Y, et al. (2014). miR-20a contributes to endometriosis by regulating NTN4 expression. Mol. Biol. Rep. 41: 5793-5797. http://dx.doi.org/10.1007/s11033-014-3452-7

Zhao X, Mohan R, Özcan S and Tang X (2012). MicroRNA-30d induces insulin transcription factor MafA and insulin production by targeting mitogen-activated protein 4 kinase 4 (MAP4K4) in pancreatic $\beta$-cells. J. Biol. Chem. 287 : 31155-31164. http://dx.doi.org/10.1074/jbc.M112.362632 